Potential factors that may influence on the relapse risk in this cohort were previously analyzed. ${ }^{51} \mathrm{~A}$ regression cox analysis including all these factors and quality indicators accomplishment was conducted. Good accomplishment of QI was defined as accomplishment of 8 or more of them.

Result(s)* A total of 82 out of the 126 participating institutions answered the survey. The final cohort was composed of 877 patients. Percentages of accomplishment of quality indicators are showed in table 1 . Two groups were defined according to the number of quality indicators accomplished (Group 1: 7 or less vs group 2: 8 or more). The rate of DFS at 4.5 year for patients who underwent surgery in centers that accomplished 7 or less quality indicators was $82.1 \%$ (78.8$85.6)$ and $89.9 \%(88.7-91.1 \%)$ in the other group. Low accomplishment of QI was associated with a lower rate of disease-free survival than High accomplishment of QI (HR, 1.61; 95\% CI, 1.01 to $2.57 ; \mathrm{P}=0.047)$. A difference that remained after adjustment for previous conization, tumour size $>2 \mathrm{~cm}$, and minimally invasive approach.

Conclusion* Good accomplishment of structural and process QI was associated with a better DFS in this European cohort.

\section{CHALLENGES IN DIAGNOSIS OF CERVICAL ADENOCARCINOMA IN A PATIENT WITH UTERINE MALFORMATION: A RARE CASE REPORT}

\footnotetext{
1;2S Álvarez Sánchez, 1;2JM Barreiro García, 1;2M Marti Sopeña, 1;2JJ Delgado Espeja, 1;2JA Solano Calvo, ${ }^{1 ; 2}$ Á Zapico Goñi. ${ }^{1}$ Hospital Universitario Príncipe de Asturias, Gynaecology, Alcalá de Henares, Spain; ${ }^{2}$ Facultad de Medicina, Universidad Alcalá de Henares, Alcalá de Henares, Spain
}

\subsection{6/ijgc-2021-ESG0.38}

Introduction/Background* Cervical cancer is the fourth most common cancer of women in the world. Some risk factors for cervical adenocarcinoma are shared with squamous cell carcinoma, mainly infection with high-risk subtypes of human papillomavirus (HPV).

Methodology A 69-year-old woman suffered recurrent postmenopausal bleeding for 2 years. Medical and surgical history: a right radical nephrectomy due to congenital renal atrophy and a partial removal of a rudimentary uterus during a c-section.

Initial medical research showed no pathology, including double liquid-based cytologies with negative result to HPV. During the follow-up period, a solid excrecent mass was discovered protruding into vagina.

Result(s)* The vaginal biopsy diagnosed a papilar adenocarcinoma of clear-cells. A computed tomography and nuclear magnetic resonance revealed a $5 \mathrm{~cm}$ solid mass located in the right sided genital system. A hysteroscopic study was also performed and revealed no lesions in vagina but a double cervix and uterus. The left-sided hemiuterus presented an atrophic endometrium, and the right-sided hemiuterus showed a proliferative endometrium. The endometrial biopsy revealed also a papilar adenocarcinoma of clear-cells with immunohistochemical study that suggested the cervical origin.

The interdisciplinary tumour committee considered the case as a cervical cancer stage IIIB, performing the paraaortic lymphadenectomy by laparoscopic surgery (negative for malignancy) and subsequently, chemoradiotherapy.

Conclusion* The difficulty of diagnosis in cervical adenocarcinoma lies in the cytology; the lack of sensitivity to adenocarcinoma may be due to these cancers developing in the endocervical canal, making sampling of abnormal cells difficult.

Clear-cell carcinoma comprises approximately $3 \%$ of all cervical adenocarcinomas and is aetiologically unrelated to HPV infection.

This case highlights some points of technical difficulties to resolve the diagnosis. First, the genital malformation that created obstacles to diagnose the origin of the pathology. Secondly, the negative result to HPV. Lastly, the unusual subtype of cervical adenocarcinoma and its predicament connected with the diagnostic sensitivity in the cytology.

\section{VARIABLES THAT PREDICT NODAL STATUS OF PATIENTS WITH EARLY CERVICAL CANCER IN THE SUCCOR COHORT}

A Berasaluce, N Martín-Calvo, E Chacon, F Boria, N Manzour, LM Chiva*. University of Navarra

\subsection{6/ijgc-2021-ESGO.39}

Introduction/Background* We aimed to identify the variables associated with higher odds of positive nodes in women in the SUCCOR study and describe both disease free survival and overall survival by nodal status in those women.

Methodology We used data from the SUCCOR study, a European multicentre study that collected retrospective information of 1272 women who underwent a radical hysterectomy by open or minimally invasive surgery for stage IB1 cervical cancer (FIGO 2009) between January 2013 and December 2014 After exclusions, the final sample included 1157 patients. Missing values were imputed with the median in quantitative variable and grouped in a new category in qualitative ones. We compared sociodemographic and clinical characteristics of patients by node status. We used Student's t test for quantitative variables and Pearson's chi squared test for qualitative ones. Variables with a $\mathrm{p}$ value below 0.05 in the univariate analyses were introduced in a logistic regression model with stepwise forward selection. Finally, we compared disease free survival and overall survival by nodal status using the LogRank test

Result(s)* We found that women with large tumours (>2 $\mathrm{cm})$, linfovascular space invasion, parametrial invasion, postoperative complications or positive (or $<2 \mathrm{~mm}$ free) margins in the tumour were more likely to have positive nodes In the multivariable adjusted model, the variables that independently predict higher odds of positive nodes were: large tumour size (OR: 1.8; 95\% CI:1.18-2.78), linfovascular space invasion (OR: 5,34; 95\% CI 3.31-8.61), parametrial invasion (OR: 5.10; 95\% CI 2.23-11.67), positive margins in the tumour (OR: 2,44; 95\% CI 1.18-5.06), and postoperative complications (OR: 2.22; 95\% CI: 1.42-3.48). The survival analyses showed significant differences in both disease-free survival $(p=0.007)$ and overall survival $(p=0,011)$ between groups.

Conclusion* In the Succor cohort the presence of large tumour size, lymphovascular space invasion, parametrial invasion, positive margins in the tumour and postoperative complications were associated with the presence of positive lymph nodes and worse survival. 